\title{
The Napkin-Ring Sign - the Story Behind Invasive Coronary Angiography
}

\author{
Zsolt Parajkó, István Kovacs, Monica Chițu, Imre Benedek \\ Center of Advanced Research in Multimodality Cardiac Imaging, Cardio Med Medical Center, Târgu Mureș, Romania
}

\section{CORRESPONDENCE \\ István Kovács \\ Str. 22 Decembrie 1989 nr. 76 \\ 540124 Târgu Mures, Romania \\ Tel: +40 265217333 \\ E-mail: kov_istvan@yahoo.com}

\section{ARTICLE HISTORY}

Received: December 12, 2020

Accepted: January 15, 2021

Published online February 18, 2021
Zsolt Parajkó • Str. 22 Decembrie 1989 nr. 76, 540124 Târgu Mureș, Romania. Tel: +40 265217 333, E-mail: parajko.zsolt@gmail.com

Monica Chițu • Str. 22 Decembrie 1989 nr. 76, 540124 Târgu Mureș, Romania. Tel: +40 265217 333, E-mail: iulia.chitu@yahoo.com

Imre Benedek • Str. 22 Decembrie 1989 nr. 76, 540124 Târgu Mureș, Romania. Tel: +40 265217333 E-mail: imrebenedek@yahoo.com

\begin{abstract}
Coronary artery disease (CAD) represents one of the leading causes of morbidity and mortality across Europe. Most of the patients do not experience any warning sign before the coronary event develops, therefore screening this group of patients is essential to prevent major cardiac events. Coronary computed tomography angiography (CCTA) offers a noninvasive approach of the coronary arteries, providing information not only on the presence and severity of the coronary stenosis, but is also able to characterize the structure of the coronary wall. CCTA allows complex evaluation of the extension of CAD, and by assessing the structure of the atherosclerotic plaque, it can identify its degree of vulnerability. The napkin-ring sign (NRS) represents a ring-like attenuation of the non-calcified portion of the coronary lesion and has a high specificity (96-100\%) for the identification of thin cap fibroatheroma (TCFA) or culprit lesion in acute coronary syndromes (ACS). It is also an independent predictor for ACS events and the strongest predictor for future ACS. Modern CCTA can provide submillimeter isotropic spatial resolution. Thus, CT attenuation-based tissue interpretation enables the assessment of total coronary plaque burden and individual plaque components, with a similar accuracy as intravascular ultrasoud-based investigations. This review aims to present the important role of CCTA as a potent screening tool for patients with CAD, and the current evidences in the detection and quantification of vulnerable plaques.
\end{abstract}

Keywords: cardiovascular diseases, napkin-ring sign, culprit lesion, vulnerable plaque

\section{INTRODUCTION}

Cardiovascular disease (CVD) is still one of the leading causes of morbidity and mortality in Europe, resulting in approximately 4 million deaths annually across the continent. The population of Eastern Europe is more likely to be affected by CVD, especially by coronary artery disease (CAD), which could progress to an acute coronary syndrome (ACS) turnout in myocardial infarction or unstable angina. ${ }^{1}$ Atherosclerosis is a chronic inflammatory process, developing macroand microstructural modifications of the artery wall, resulting in CVD. Lipid accumulation in the arterial intima, inflammation (local inflammatory reaction and macrophage infiltration of the affected arterial segment), foam cell formation, muscle cell proliferation, necrosis, calcification, and arterial wall fibrosis induce important functional and morphological changes and lead to plaque formation, 
resulting in $\mathrm{CAD} .^{2}$ The progression of $\mathrm{CAD}$ varies among patients, but generally it takes a long period of time until the first clinical manifestation of the disease appears. ${ }^{3}$ The most common and dramatic manifestation of CAD is related to plaque rupture or erosion, followed by the activation of the coagulation cascade and subsequently intraluminal thrombosis. In a significant part of the cases, the first manifestation of CAD is acute myocardial infarction (AMI) or sudden cardiac death (in $50 \%$ of men and $64 \%$ of women). ${ }^{4}$ Therefore, most of the patients presenting with ACS do not experience any symptoms or warning sings before the coronary event occurs. There are several methods to evaluate patients with stable chest pain. Coronary computed tomography angiography (CCTA) offers a noninvasive approach of the coronary arteries, being recommended as a screening tool, especially for patients with low to intermediate risk of CAD. ${ }^{5}$ Conventional/invasive coronary angiography - ICA (considered the gold standard for the diagnosis of significant $\mathrm{CAD}$ ) has a high specificity and sensibility to assess the obstructive lesions, but it is unable to characterize the plaque structure or to identify the high risk plaques, although it was demonstrated by several studies that a notable proportion of acute coronary events occur due to the rupture of mildly stenotic plaques. ${ }^{6,7}$ Compared to conventional coronarography, CCTA can provide prognostic information from non-obstructive lesions by identifying vulnerable plaque parameters such as the napkin-ring sign, low attenuation, spotty calcification, and positive remodeling of the vessel. Moreover, non-contrast CT scans can assess epicardial adipose tissue (EAT) and by quantifying coronary artery calcification, using a score system (Agatson score), they can predict plaque progression and future coronary events beyond traditional risk factors. ${ }^{8,9}$

\section{BEYOND THE CORONARY PLAQUE MORPHOLOGY}

Plaque rupture, followed by an ACS, has been related to the rupture or erosion of a vulnerable plaque with a lipidrich necrotic core and a thin fibrous cap, followed by acute thrombosis and subsequently coronary occlusion. ${ }^{7,10}$

Currently, there are six types of atherosclerotic lesions, defined by the American Heart Association (AHA). Type I - initial lesion, characterized by intimal thickening and smooth muscle cell accumulation in the intima; Type II "fatty streak" with luminal accumulation of foam cells, it usually regresses; Type III - preatheroma or progressive atherosclerotic lesion with extracellular lipid accumulation and pathologic intimal thickening; Type IV - atheroma; Type V - fibroatheroma, with thin fibrous cap, infiltrated by macrophages and lymphocytes, with the presence of a necrotic core; Type VI - complicated plaque, without intact plaque surface and/or hematoma/hemorrhage and/ or thrombotic deposit. ${ }^{11}$ Based on the AHA classification, type I and type II plaques are reversible, capable to regress or to progress. Type III lesions are considered precursors of the fibroatheroma, described by pathological structural changes such as an escalated amounts of lipids and multiple smooth muscle cell layers near the lumen. These "proatheromas" are infiltrated with a great number of foam cells. Interestingly, $40 \%$ of these cells originate from smooth muscle cells and $60 \%$ from macrophages. ${ }^{12,13}$ The progression of deep intimal thickening to pathologic intimal thickening depends on a complex interaction between retention and oxidation of lipids, as well as smooth muscle cell proliferation and inflammation. ${ }^{13}$ Type IV lesions represent the first stage of advanced plaques, characterized by the appearance of the fibrous cap (containing collagen and proteoglycans, smooth muscle cells, macrophages, and lymphocytes), framing the necrotic core (with large extent of lipids, originating from extracellular lipid pools and insufficient efferocytosis of dead smooth muscle cells and macrophages). The evolved foam cells in this region will be damaged (mostly by apoptosis), subsequently releasing further inflammatory material. ${ }^{14,15}$ From this stage of lesions, they can cause significant luminal narrowing and can progress to complicated plaque with surface erosion, thrombosis, and calcification. Based on the thickness of the fibrous cap, they are less or more prone to complication. Numerous studies showed that the fibrous cap thickness (TCAP) represents one of the best markers for vulnerable plaques, a cap thickness between $54-84 \mu \mathrm{m}$ being considered a vulnerable, high-risk plaque. ${ }^{16}$ Postmortem assessment of coronary arteries, by examination of coronary cross-section layers, showed that $95 \%$ of ruptured plaques had a thickness of the fibrous cap lower than $65 \mu \mathrm{m} .{ }^{17}$ Optical coherence tomography (OCT) examination in patients with ACS determined a median cap thickness of $80 \mu \mathrm{m}$ in non-ruptured plaques. ${ }^{16}$ Without an intact fibrous cap, the lipid-rich necrotic core can interact with the blood flow, facilitating platelet accumulation and thrombus formation in the affected segments. Histopathological partition distinguished three types of lesions prone to provoke acute coronary events: rupture, erosion, and calcified nodule. ${ }^{18}$

Ruptured plaques are characterized by a lipid-rich necrotic core, surrounded by a thin, fibrous cap with active inflammation. In most cases, the appearance of luminal thrombosis is caused by plaque rupture (approximatively $60-70 \%)$, the rest of the cases resulting from plaque erosion, and about $5 \%$ being caused by thrombus formation 
at the level of calcified nodules. ${ }^{19}$ Plaque erosion is defined by a relatively intact intima, without cap disruption, where the blood flow comes into direct contact with the intimal surface due to lack of endothelial cells, favorizing thrombus formation..$^{20}$ Interestingly, autopsies revealed that a remarkable part of the thrombi in erosion (more than $85 \%$ ) showed evidence of a healing process (acute inflammatory cell lysis, invasion by smooth muscle cells, organized layers of smooth muscle cells, proteoglycans with varying degrees of platelet/fibrin layering). On the other hand, a healing process was observed only in half of the ruptured plaques. ${ }^{21}$ The examination of coronary cross-sections suggested that repeated plaque ruptures can initiate further plaque progression. ${ }^{22}$

The extension of the necrotic core also correlates with plaque complication. As the necrotic core extends outwards, causing thickening of the fibrous cap, concomitantly smooth muscle cells are depleted from the fibrous cap through cell apoptosis, therefore the cap thickness decreases. ${ }^{23,24} \mathrm{Si}-$ multaneously with plaque progression, the vasa vasorum infiltrates the lesion and becomes leaky, provoking intraplaque hemorrhage. ${ }^{25}$ The number of smooth muscle cells is inversely correlated with the progression of the atheroma because of migration and cell apoptosis. ${ }^{26}$ There are numerous factors that induce smooth muscle cell apoptosis, thus the contribution of progressive cell loss to plaque stability is more complex and strongly affects the local milieu. ${ }^{27,28}$ This milieu in atherosclerotic lesions inhibits the phagocytosis and defective efferocytosis of apoptotic cells, followed by secondary necrosis and leakage of intracellular contents, maintaining and exacerbating the inflammatory milieu. ${ }^{29}$

In conclusion, there are several morphological and functional changes that lead to the progression of a preatheroma to a vulnerable plaque. Vulnerable plaques prone to rupture contain a large necrotic core, active inflammation, and macrophage infiltration, a thin fibrous cap, and numerous vasa vasorum at the affected level. ${ }^{30}$ The mechanisms of the rupture of a thin cap fibroatheroma are unclarified yet. Increased proteolytic activity, mechanical stress, and microcalcification of the fibrous cap are considered to be relevant factors. ${ }^{31-34}$

\section{CAN WE PREVENT ACUTE MYOCARDIAL INFARCTION BY SCREENING FOR CORONARY ARTERY DISEASE?}

Prevention of acute coronary events seems to be the only potent strategy to reduce the rate of major acute cardiovascular events (MACE) and improve mortality and morbidity. ${ }^{35}$ Although major efforts are ongoing to predict which plaque will cause MACE on an individual plaque level, currently the diagnostic strategies target the detection of myocardial ischemia and hemodynamically significant luminal narrowing, but ignore the huge issue of a MACE occurring as the first (in half of the individuals) and only manifestation of CAD.

Based on the morphological characteristics of the fibroatheroma, there are several imaging features that aid the identification of vulnerable plaques. The rapid progression and development of cardiovascular imaging technologies in recent decades has enabled the introduction of several screening imaging procedures into clinical practice for the primary prevention or assessment of the extension of CAD, which could be useful to detect vulnerable lesions. However, in spite of these major developments, identifying vulnerable plaques remains a major challenge. The early diagnosis of CAD could promote physicians to initiate complex treatment for adequate primary prevention, thus reducing the incidence of acute coronary events. ${ }^{36}$ There are several imaging methods to assess the coronary vessels, ICA being considered the gold standard for the diagnosis of CAD (both stable and ACS). ${ }^{37}$ Although ICA can describe the anatomy of the coronary vessels and quantify the lumennarrowing stenosis, it does not provide information about the wall structure of the coronary vessel or the composition of the atherosclerotic plaque, therefore being unable to identify the vulnerable plaques. ${ }^{38}$ Additional invasive imaging methods could be used for a better evaluation of the extension of CAD and to identify the presence of a rupture-prone plaque. OCT can provide very high resolution $(10-20 \mu \mathrm{m})$ images and is able to do a microscopic characterization of plaque morphology, therefore it can be used to evaluate plaque vulnerability by measuring the thickness of the fibrous cap, and to detect lipid content. Moreover, only OCT can detect the eroded plaques. ${ }^{39}$

Intravascular ultrasound (IVUS) represents another invasive intravascular imaging technique to improve the diagnostic accuracy of ICA. IVUS could be used for measuring the lumen area, plaque burden, and vascular remodeling. IVUS-based studies identified several imaging biomarkers in association with plaque vulnerability, such as the presence of an extensive necrotic core surrounded by a thin fibrous cap, with dense macrophage infiltration, large lipid pool, spotty calcification, and positive vascular remodeling. ${ }^{40,41}$ Spectroscopy, thermography, angioscopy, and intravascular cardiac magnetic resonance also represent invasive imaging methods that could be used to provide additional information such as plaque temperature, plaque inflammation, and neoangiogenesis.

Although the previously presented invasive imaging markers could identify and precisely describe the 
high-risk atherosclerotic plaques, they cannot be used as a screening method for primary prevention in a large population. Patel et al. found that among 398,978 elective invasive coronary angiographies, only $38 \%$ of the cases were positive for obstructive $\mathrm{CAD}, 62 \%$ of the patients had no obstructive disease, and $39 \%$ had normal coronary arteries. ${ }^{42}$ Cardiac CCTA offers a noninvasive approach for CAD patients and can used as a "gatekeeper" to reduce unnecessary invasive diagnostic procedures. Using at least 64-slice multidetector row has a sensibility of $98 \%$ and a specificity of $90 \%$. Due to the elevated sensitivity, CCTA investigations have a 95 to $100 \%$ negative predictive value to rule out obstructive CAD and an excellent negative predictive value for future events. ${ }^{43}$ Multidetector computed tomography (MDCT) has the ability to describe and accurately localize the lumen-narrowing atherosclerotic plaques. By measuring local tissue attenuation, MDCT can assess the morphology and composition of these lesions with high temporal and spatial resolution. Moreover, in the guidelines for the diagnosis and management of chronic coronary syndrome published by the European Society of Cardiology, computed tomography angiography has a class I recommendation for diagnosing CAD in symptomatic patients. ${ }^{44}$ Contrastenhanced ultrasonography and high-resolution magnetic resonance imaging are also noninvasive investigations to characterize the coronary lesions, within their benefits and limits.

Modern multislice computed tomography (MSCT) with increased spatial resolution permits a more complex characterization of non-calcified coronary plaques (defined as $<130$ Hounsfield units), assessing positive remodeling, low attenuation plaque, and spotty calcification. ${ }^{45}$ Moreover, on CCTA images, several authors described a ring-like attenuation of the non-calcified portion of the coronary lesion, which was termed the napkin-ring sign. ${ }^{46,47}$ Thus, non-calcified plaques are categorized into three different classes: homogenous plaque, non-napkin-ring sign heterogenous plaques, and napkin-ring sign heterogenous plaques. ${ }^{48}$ The napkin-ring sign represents a large necrotic core - low attenuation core, surrounded by a thin-cap fibroatheroma - rim-like thin area of higher attenuation. Histopathological analyses on ex vivo donor heart demonstrated the correspondence between the napkin-ring sign and the presence of histological markers of a vulnerable plaque at that sequence. ${ }^{49}$ Based on recent studies, besides the napkin-ring sign, the only independent predictors for future MACE were representsed by fibrofatty plaque features, which were associated with events in short- and mid-term outcome periods. ${ }^{50-52}$

\section{THE NAPKIN-RING SIGN}

As we mentioned before, the histopathological analysis of culprit and non-culprit thin-cap fibroatheromas (TCFAs) with identical luminal narrowing effect found that only fibrous cap thickness and necrotic core size are independent predictors of plaque rupture. ${ }^{53}$ Furthermore, a large necrotic core cross-sectional area and the presence of macrophage infiltration are the best discriminators between a ruptured plaque or TCFAs and a stable atherosclerotic lesion..$^{10}$ Moreover, in $80 \%$ of the vulnerable plaques prone to rupture, the necrotic core area is $>1$ $\mathrm{mm}^{2}{ }^{24}$ Modern CCTA can provide submillimeter isotropic spatial resolution, thus the $\mathrm{CT}$ attenuation-based tissue interpretation enables the assessment of total coronary plaque burden and individual plaque components, with a similar accuracy as IVUS-based investigations. 7,55

A ring-like $\mathrm{CT}$ attenuation pattern of non-calcified plaque was present in ACS culprit lesions and in plaques associated with slow-flow or no-reflow phenomenon during ICA. 56,57 There are two major qualitive components in the CT images of a vulnerable non-calcified plaque which fulfill the criteria of a napkin-ring sign: a center area of low CT attenuation tissue, apparently in contact with the lumen, and a higher attenuation plaque segment surrounding this central area (fibrous plaque tissue). ${ }^{48,49,58}$ The necrotic core area represents an important factor compared with nonnapkin-ring sign plaques, as it can be over twice the size in napkin-ring sign plaques (median $1.1 \mathrm{~mm}^{2}$ versus 0.46 $\left.\mathrm{mm}^{2}\right){ }^{58}$ Interestingly, the majority of these high-risk lesions have a necrotic core area over $1.0 \mathrm{~mm}^{2} .{ }^{30}$ Based on CT images, the specificity of a napkin-ring sign for a vulnerable atherosclerotic lesion and TCFA is exceptional (98\% and $94.1 \%$ ), but unfortunately its specificity is relatively low. 48,59 In clinical investigations, the napkin-ring sign had a high specificity (96-100\%) for the identification of TCFA or culprit ACS, being also an independent predictor for ACS events. ${ }^{46,60}$ Studies also found that the napkin-ring sign was more frequent in TCFA compared with non-TCFA plaques (controlled by OCT). ${ }^{56}$ Otsuka et al. found that the presence of the napkin-ring sign was the strongest predictor for future ACS [HR 5.55; 95\% CI 2.10-14.70, p <0.001], independent of other high-risk criteria. ${ }^{46}$ Furthermore, a longterm follow-up study of 7.8 years in a group of patients with CAD (including low-to-intermediate risk) evidentiated an increased prevalence of the napkin-ring sign in patients with adverse events $(63.4 \%$ vs. $2.8 \%, \mathrm{p}<0.001)$. Moreover, the napkin-ring sign was the most important predictor of adverse events after the assessment of the conventional risk factors, stenosis severity, and plaque type. ${ }^{61}$ 


\section{THE POTENTIAL UTILITY OF CCTA AND THE CLINICAL IMPLICATIONS OF THE NAPKIN-RING SIGN}

The constant upgrade of MSCT technology and new software features allow a more complex and more detailed noninvasive plaque characterization using morphological and functional data (CT-FFR and ESS). Combining morphological and functional characteristics of the atherosclerotic plaque results in a highly accurate detection of the vulnerable and stable lesions. This new feature has established CCTA as a first-line coronary assessment method in patients with low to intermediate risk of CAD. ${ }^{44,62} \mathrm{In}$ addition, the presence of inflammation at the coronary lesion could favor the atherosclerotic process. Given its use in the complex assessment of the perivascular adipose tissue, recently CCTA has been proposed as an effective tool for the quantification of coronary inflammation, which could be an important factor-besides the morphological and functional assessment of the coronary plaque-for an individualized coronary risk stratification. ${ }^{63,64}$

Using CCTA as a potential screening tool for low-tointermediate risk of CAD could significantly reduce the unnecessary ICA; moreover, CCTA has an excellent negative predictive value and could be a good alternative to rule out the presence of CAD when pretest probability is intermediate. ${ }^{65}$ In the PROspective Multicenter Imaging Study for Evaluation of chest pain (PROMISE) trial, which compared the presence of CAD in patients with typical symptoms evaluated with CCTA versus functional testing, the rate of non-CAD was $28 \%$ in the CCTA group and $52 \%$ in the functional testing group. ${ }^{66}$ Despite the availability of CCTA or functional testing, up to $45 \%$ of patients are directly referred for ICA without prior functional testing. ${ }^{67} \mathrm{It}$ is important to mention that although the technological improvement and the widely used radial access have reduced the complication rate of ICA, approximately $0.6-4.2 \%$ of the procedures have different type of complications. ${ }^{68}$

Based on the previously cited studies, CCTA can provide accurate characterization of the coronary arteries by detecting the obstructive plaques, and through its ability to visualize the coronary artery wall it could be used to assess plaque morphology. Thus, CCTA images could help to identify high-risk plaques, and by using a noninvasive imaging method the physician could initiate the adequate treatment. Moreover, CCTA may help to identify coronary artery lesion progression or regression, providing a good feedback and control of the chosen therapy. ${ }^{69,70}$ Although it is not possible to control the therapeutic efficiency of a chosen intervention on the atherosclerotic plaque, repeated
CCTA examinations with direct monitorization of plaque progression may lead to a superior therapeutic modulation. ${ }^{71}$

Another revolutionary perspective of CCTA is the use of radiomics, a process where numerous quantitative features are extracted from the investigated region to create large data sets in which each abnormality is described by hundreds of parameters. The applicability of these features is still at an early stage, but the rapid progress encountered in the field of artificial intelligence will probably bring major changes in the way we integrate clinical, biological, and imaging information for a complex, individualized risk stratification and therapeutic decision-making. ${ }^{72}$

\section{CONCLUSION}

The evolution and rapid progression of CT imaging technologies has changed the therapeutic approach of patients with suspected CAD, offering an early and accurate assessment of the coronary arteries. Due to the capability of CCTA to identify multiple high-risk features of atherosclerotic plaques, such as the napkin-ring sign, positive remodeling, low CT attenuation, low endothelial shear stress, and spotty calcification, this modern imaging technique may improve clinical evolution and lead to a more effective management of the cardiovascular patient.

\section{CONFLICT OF INTEREST}

Nothing to declare.

\section{ACKNOWLEDGEMENT}

This research was supported via the research grant no. 103544/2016, contract number 26/01.09.2016, entitled "Increasing the research capacity in the field of vulnerable plaque imaging, based on advanced nanoparticles, fusion imaging and computational simulation - PlaqueImage", financed by the Romanian Ministry of European Funds, the Romanian Government and the European Union.

\section{REFERENCES}

1. Townsend N, Wilson L, Bhatnagar P, Wickramasinghe K, Rayner M, Nichols M. Cardiovascular disease in Europe: epidemiological update 2016. Eur Heart J. 2016:37:3232-3245.

2. Mushenkova NV, Summerhill VI, Zhang D, Romanenko EB, Grechko AV, Orekhov AN. Current Advances in the Diagnostic Imaging of Atherosclerosis: Insights into the Pathophysiology of Vulnerable Plaque. Int J Mol Sci. 2020;21:2992.

3. Eikendal ALM, Groenewegen KA, Bots ML, Peters SAE, Uiterwaal CSPM, den Ruijter HM. Relation Between Adolescent Cardiovascular Risk Factors and Carotid Intima-Media Echogenicity in Healthy Young Adults: The 
Atherosclerosis Risk in Young Adults (ARYA) Study. J Am Heart Assoc. 2016;5: e002941.

4. Libby P. Mechanisms of Acute Coronary Syndromes and Their Implications for Therapy. N Engl J Med. 2013;368:2004-2013.

5. The SCOT-HEART Investigators. Coronary CT Angiography and 5-Year Risk of Myocardial Infarction. N Engl J Med. 2018;379:924-933.

6. Glaser R, Selzer F, Faxon DP, et al. Clinical Progression of Incidental, Asymptomatic Lesions Discovered During Culprit Vessel Coronary Intervention. Circulation. 2005;111:143-149.

7. Stone GW, Maehara A, Lansky AJ, et al. A Prospective Natural-History Study of Coronary Atherosclerosis. N Engl J Med. 2011;364:226-235.

8. Pugliese G, lacobini C, Fantauzzi CB, Menini S. The dark and bright side of atherosclerotic calcification. Atherosclerosis. 2015;238:220-230.

9. Park J-S, Choi S-Y, Zheng M, et al. Epicardial adipose tissue thickness is a predictor for plaque vulnerability in patients with significant coronary artery disease. Atherosclerosis. 2013;226:134-139.

10. Narula J, Nakano M, Virmani R, et al. Histopathologic Characteristics of Atherosclerotic Coronary Disease and Implications of the Findings for the Invasive and Noninvasive Detection of Vulnerable Plaques. J Am Coll Cardiol. 2013;61:1041-1051.

11. Virmani R, Kolodgie FD, Burke AP, Farb A, Schwartz SM. Lessons From Sudden Coronary Death: A Comprehensive Morphological Classification Scheme for Atherosclerotic Lesions. Arterioscler Thromb Vasc Biol. 2000;20:1262-1275.

12. Boucher P, Matz RL, Terrand J. atherosclerosis: gone with the Wnt? Atherosclerosis. 2020;301:15-22.

13. Basatemur GL, Jørgensen HF, Clarke MCH, Bennett MR, Mallat Z. Vascular smooth muscle cells in atherosclerosis. Nat Rev Cardiol. 2019;16:727-744.

14. Clarke MCH, Talib S, Figg NL, Bennett MR. Vascular Smooth Muscle Cell Apoptosis Induces Interleukin-1-Directed Inflammation: Effects of Hyperlipidemia-Mediated Inhibition of Phagocytosis. Circ Res. 2010;106:363-372.

15. Nakashima Y, Wight TN, Sueishi K. Early atherosclerosis in humans: role of diffuse intimal thickening and extracellular matrix proteoglycans. Cardiovasc Res. 2008;79:14-23.

16. Yonetsu T, Kakuta T, Lee $\mathrm{T}$, et al. In vivo critical fibrous cap thickness for rupture-prone coronary plaques assessed by optical coherence tomography. Eur Heart J. 2011;32:1251-1259.

17. Burke AP, Farb A, Malcom GT, Liang Y, Smialek J, Virmani R. Coronary Risk Factors and Plaque Morphology in Men with Coronary Disease Who Died Suddenly. N Engl J Med. 1997;336:1276-1282.

18. Arbustini E, Bello BD, Morbini P, et al. Plaque erosion is a major substrate for coronary thrombosis in acute myocardial infarction. Heart. 1999;82:269-272

19. van der Wal AC, Becker AE, van der Loos CM, Das PK. Site of intimal rupture or erosion of thrombosed coronary atherosclerotic plaques is characterized by an inflammatory process irrespective of the dominant plaque morphology. Circulation. 1994;89:36-44.

20. Jinnouchi $H$, Virmani R, Finn AV. Are characteristics of plaque erosion defined by optical coherence tomography similar to true erosion in pathology? Eur Heart J. 2018;39:2086-2089

21. Kramer MCA, Rittersma SZH, de Winter RJ, et al. Relationship of Thrombus Healing to Underlying Plaque Morphology in Sudden Coronary Death. $J$ Am Coll Cardiol. 2010;55:122-132.

22. Mann J, Davies MJ. Mechanisms of progression in native coronary artery disease: role of healed plaque disruption. Heart. 1999;82:265-268.

23. Falk E. Plaque rupture with severe pre-existing stenosis precipitating coronary thrombosis. Characteristics of coronary atherosclerotic plaques underlying fatal occlusive thrombi. Br Heart J. 1983;50:127-134.

24. Kolodgie FD, Fowler DR, Farb A, Narula J. Intraplaque Hemorrhage and Progression of Coronary Atheroma. N Engl J Med. 2003:10:2316-2325.

25. Sluimer JC, Kolodgie FD, Bijnens APJJ, et al. Thin-Walled Microvessels in Human Coronary Atherosclerotic Plaques Show Incomplete Endothelial Junctions. J Am Coll Cardiol. 2009;53:1517-1527

26. Davies MJ, Richardson PD, Woolf N, Katz DR, Mann J. Risk of thrombosis in human atherosclerotic plaques: role of extracellular lipid, macrophage, and smooth muscle cell content. Heart. 1993;69:377-381.

27. Proudfoot D, Skepper JN, Hegyi L, Bennett MR, Shanahan CM, Weissberg PL. Apoptosis Regulates Human Vascular Calcification In Vitro: Evidence for Initiation of Vascular Calcification by Apoptotic Bodies. Circ Res. 2000;87:1055-1062.

28. Patel VA, Zhang Q-J, Siddle K, et al. Defect in Insulin-Like Growth Factor-1 Survival Mechanism in Atherosclerotic Plaque-Derived Vascular Smooth Muscle Cells Is Mediated by Reduced Surface Binding and Signaling. Circ Res. 2001;88:895-902.
29. Ait-Oufella H, Pouresmail V, Simon T, et al. Defective Mer Receptor Tyrosine Kinase Signaling in Bone Marrow Cells Promotes Apoptotic Cell Accumulation and Accelerates Atherosclerosis. Arterioscler Thromb Vasc Biol. 2008;28:1429-1431.

30. Virmani R, Burke AP, Farb A, Kolodgie FD. Pathology of the Vulnerable Plaque. J Am Coll Cardiol. 2006;47:C13-C18.

31. Hutcheson JD, Goettsch C, Bertazzo S, et al. Genesis and growth of extracellular-vesicle-derived microcalcification in atherosclerotic plaques. Nat Mater. 2016;15:335-343.

32. Gijsen F, van der Giessen A, van der Steen A, Wentzel J. Shear stress and advanced atherosclerosis in human coronary arteries. J Biomech. 2013:46:240-247.

33. Yu H, Fellows A, Foote K, et al. FOXO3a (Forkhead Transcription Factor O Subfamily Member 3a) Links Vascular Smooth Muscle Cell Apoptosis, Matrix Breakdown, Atherosclerosis, and Vascular Remodeling Through a Novel Pathway Involving MMP13 (Matrix Metalloproteinase 13). Arterioscler Thromb Vasc Biol. 2018;38:555-565.

34. Vengrenyuk Y, Carlier S, Xanthos S, et al. A hypothesis for vulnerable plaque rupture due to stress-induced debonding around cellular microcalcifications in thin fibrous caps. Proc Natl Acad Sci. 2006;103:1467814683.

35. Pursnani A, Schlett CL, Mayrhofer T, et al. Potential for coronary CT angiography to tailor medical therapy beyond preventive guidelinebased recommendations: Insights from the ROMICAT I trial. J Cardiovasc Comput Tomogr. 2015;9:193-201.

36. Honigberg MC, Lander BS, Baliyan V et al. Preventive Management of Nonobstructive CAD After Coronary CT Angiography in the Emergency Department. JACC Cardiovasc Imaging. 2020;13:437-448.

37. Chieffo A, Giustino G, Spagnolo P, et al. Routine Screening of Coronary Artery Disease With Computed Tomographic Coronary Angiography in Place of Invasive Coronary Angiography in Patients Undergoing Transcatheter Aortic Valve Replacement. Circ Cardiovasc Interv. 2015;8: e002025.

38. Julio Nunez GM. Coronary Angiography, Too Far to be a Gold Standard Technique for Identifying a Vulnerable Plaque. Journal of Clinical \& Experimental Cardiology. 2011;02:1000132.

39. Tearney GJ, Regar E, Akasaka T, et al. Consensus Standards for Acquisition, Measurement, and Reporting of Intravascular Optical Coherence Tomography Studies. J Am Coll Cardiol. 2012;59:1058-1072.

40. Hong YJ, Jeong MH, Choi YH, et al. Comparison of Coronary Plaque Components between Non-Culprit Lesions in Patients with Acute Coronary Syndrome and Target Lesions in Patients with Stable Angina: Virtual Histology-Intravascular Ultrasound Analysis. Korean Circ J. 2013;43:607.

41. Hong M-K, Mintz GS, Lee CW, et al. Comparison of Virtual Histology to Intravascular Ultrasound of Culprit Coronary Lesions in Acute Coronary Syndrome and Target Coronary Lesions in Stable Angina Pectoris. Am J Cardiol. 2007:100:953-959.

42. Rubin GD. Emerging and Evolving Roles for CT in Screening for Coronary Heart Disease. J Am Coll Radiol. 2013;10:943-948.

43. Pontone G, Bertella E, Mushtaq S, et al. Coronary Artery Disease: Diagnostic Accuracy of CT Coronary Angiography-A Comparison of High and Standard Spatial Resolution Scanning. Radiology. 2014;271:688-694.

44. Knuuti J. 2019 ESC Guidelines for the diagnosis and management of chronic coronary syndromes The Task Force for the diagnosis and management of chronic coronary syndromes of the European Society of Cardiology (ESC). Russ J Cardiol. 2020;25:119-180

45. Thomsen C, Abdulla J. Characteristics of high-risk coronary plaques identified by computed tomographic angiography and associated prognosis: a systematic review and meta-analysis. Eur Heart J Cardiovasc Imaging. 2016;17:120-129.

46. Otsuka K, Fukuda S, Tanaka A, et al. Napkin-Ring Sign on Coronary CT Angiography for the Prediction of Acute Coronary Syndrome. JACC Cardiovasc Imaging. 2013;6:448-457.

47. Kolossváry M, Karády J, Szilveszter B, et al. Radiomic Features Are Superior to Conventional Quantitative Computed Tomographic Metrics to Identify Coronary Plaques With Napkin-Ring Sign. Circ Cardiovasc Imaging. 2017;10:e006843.

48. Maurovich-Horvat P, Schlett CL, Alkadhi H, et al. The Napkin-Ring Sign Indicates Advanced Atherosclerotic Lesions in Coronary CT Angiography. JACC Cardiovasc Imaging. 2012;5:1243-1252.

49. Maurovich-Horvat P, Hoffmann U, Vorpahl M, Nakano M, Virmani R, Alkadhi $H$. The Napkin-Ring Sign: CT Signature of High-Risk Coronary Plaques? JACC Cardiovasc Imaging. 2010;3:440-444.

50. Williams MC, Moss AJ, Dweck M, et al. Coronary Artery Plaque Characteristics Associated With Adverse Outcomes in the SCOT-HEART Study. J Am Coll Cardiol. 2019;73:291-301. 
51. Ferencik M, Mayrhofer T, Bittner DO, et al. Use of High-Risk Coronary Atherosclerotic Plaque Detection for Risk Stratification of Patients With Stable Chest Pain: A Secondary Analysis of the PROMISE Randomized Clinical Trial. JAMA Cardiol. 2018;3:144

52. Senoner T, Plank F, Barbieri F, et al. Added value of high-risk plaque criteria by coronary CTA for prediction of long-term outcomes. Atherosclerosis. 2020;300:26-33.

53. Finn AV, Nakano M, Narula J, Kolodgie FD, Virmani R. Concept of Vulnerable/ Unstable Plaque. Arterioscler Thromb Vasc Biol. 2010;30:1282-1292.

54. Celeng C, Takx RAP, Ferencik M, Maurovich-Horvat P. Non-invasive and invasive imaging of vulnerable coronary plaque. Trends Cardiovasc Med. 2016;26:538-547.

55. Schepis T, Marwan M, Pflederer T, et al. Quantification of non-calcified coronary atherosclerotic plaques with dual-source computed tomography: comparison with intravascular ultrasound. Heart. 2010;96:610-615.

56. Ito T, Terashima M, Kaneda H, et al. Comparison of In Vivo Assessment of Vulnerable Plaque by 64-Slice Multislice Computed Tomography Versus Optical Coherence Tomography. Am J Cardiol. 2011;107:1270-1277.

57. Kodama T, Kondo T, Oida A, Fujimoto S, Narula J. Computed Tomographic Angiography-Verified Plaque Characteristics and Slow-Flow Phenomenon During Percutaneous Coronary Intervention. JACC Cardiovasc Interv. 2012;5:636-643.

58. Seifarth H, Schlett CL, Nakano M, et al. Histopathological correlates of the napkin-ring sign plaque in coronary CT angiography. Atherosclerosis. 2012;224:90-96

59. Maurovich-Horvat P, Ferencik M, Voros S, Merkely B, Hoffmann U. Comprehensive plaque assessment by coronary CT angiography. Nat Rev Cardiol. 2014:11:390-402.

60. Motoyama S, Sarai M, Harigaya H, et al. Computed Tomographic Angiography Characteristics of Atherosclerotic Plaques Subsequently Resulting in Acute Coronary Syndrome. J Am Coll Cardiol. 2009;54:49-57.

61. Feuchtner $G$, Kerber J, Burghard P, et al. The high-risk criteria low attenuation plaque $<60 \mathrm{HU}$ and the napkin-ring sign are the most powerfu predictors of MACE: a long-term follow-up study. Eur Heart J - Cardiovasc Imaging. 2017;18:772-779

62. Moss AJ, Williams MC, Newby DE, Nicol ED. The Updated NICE Guidelines: Cardiac CT as the First-Line Test for Coronary Artery Disease. Curr Cardiovasc Imaging Rep. 2017;10:15.

63. Antoniades C, Kotanidis CP, Berman DS. State-of-the-art review article Atherosclerosis affecting fat: What can we learn by imaging perivascular adipose tissue? J Cardiovasc Comput Tomogr. 2019;13:288-296.
64. Oikonomou EK, West HW, Antoniades C. Cardiac Computed Tomography: Assessment of Coronary Inflammation and Other Plaque Features. Arterioscler Thromb Vasc Biol. 2019;39:2207-2219.

65. Houssany-Pissot S, Rosencher J, Allouch P, et al. Screening coronary artery disease with computed tomography angiogram should limit normal invasive coronary angiogram, regardless of pretest probability. Am Heart J. 2020;223:113-119.

66. Douglas PS, Hoffmann U, Patel MR, et al. Outcomes of Anatomical versus Functional Testing for Coronary Artery Disease. N Engl J Med. 2015;372(14):1291-1300.

67. Lin GA, Dudley RA, Lucas FL, Malenka DJ, Vittinghoff E, Redberg RF. Frequency of Stress Testing to Document Ischemia Prior to Elective Percutaneous Coronary Intervention. JAMA. 2008;300:1765-1773.

68. Nakazato R, Arsanjani R, Achenbach S, et al. Age-related risk of major adverse cardiac event risk and coronary artery disease extent and severity by coronary CT angiography: results from 15187 patients from the International Multisite CONFIRM Study. Eur Heart J - Cardiovasc Imaging. 2014;15:586-594.

69. Giannopoulos AA, Mitsouras D, Bartykowszki A, et al. High-Risk Plaque Regression and Stabilization: Hybrid Noninvasive Morphological and Hemodynamic Assessment. Circ Cardiovasc Imaging. 2018;11:e007888.

70. Shi R, Shi K, Yang Z, et al. Serial coronary computed tomography angiography-verified coronary plaque progression: comparison of stented patients with or without diabetes. Cardiovasc Diabetol. 2019;18:123.

71. Lee S-E, Chang H-J, Rizvi A, et al. Rationale and design of the Progression of AtheRosclerotic PIAque Determlned by Computed TomoGraphic Angiography IMaging (PARADIGM) registry: A comprehensive exploration of plaque progression and its impact on clinical outcomes from a multicenter serial coronary computed tomographic angiography study. Am Heart J. 2016;182:72-79.

72. Opincariu D, Benedek T, Chițu M, Raț N, Benedek I. From CT to artificial intelligence for complex assessment of plaque-associated risk. Int J Cardiovasc Imaging. 2020;36:2403-2427. 